PHYSICAL REVIEW D 89, 023503 (2014)

\title{
Null tests of the cosmological constant using supernovae
}

\author{
Sahba Yahya, ${ }^{1}$ Marina Seikel, ${ }^{1}$ Chris Clarkson, ${ }^{2}$ Roy Maartens, ${ }^{1,3}$ and Mathew Smith ${ }^{1}$ \\ ${ }^{1}$ Physics Department, University of the Western Cape, Cape Town 7535, South Africa \\ ${ }^{2}$ Department of Mathematics and Applied Mathematics, University of Cape Town, \\ Cape Town 7701, South Africa \\ ${ }^{3}$ Institute of Cosmology and Gravitation, University of Portsmouth, Portsmouth PO1 3FX, United Kingdom
}

(Received 15 October 2013; published 9 January 2014)

\begin{abstract}
The standard concordance model of the Universe is based on the cosmological constant as the driver of accelerating expansion. This concordance model is being subjected to a growing range of interlocking observations. In addition to using generic observational tests, one can also design tests that target the specific properties of the cosmological constant. These null tests do not rely on parametrizations of observables, but focus on quantities that are constant only if dark energy is a cosmological constant. We use supernova data in null tests that are based on the luminosity distance. In order to extract derivatives of the distance in a model-independent way, we use Gaussian processes. We find that the concordance model is compatible with the Union 2.1 data, but the error bars are fairly large. Simulated data sets are generated for the Dark Energy Survey supernova survey and we show that this survey will allow for a sharper null test of the cosmological constant if we assume the Universe is flat. Allowing for spatial curvature degrades the power of the null test.
\end{abstract}

DOI: $10.1103 /$ PhysRevD.89.023503

PACS numbers: 98.80.-k, 98.80.Es, 95.36.+x

\section{INTRODUCTION}

The simplest model that can explain the apparent acceleration of the Universe is the "concordance" $\Lambda \mathrm{CDM}$ model, with $\Omega_{m} \approx 0.3$ and zero spatial curvature $\Omega_{K}=0$. The concordance model is consistent with all observations to date [1]. Current observations favor a dark energy model with equation of state $w(z) \approx-1$, although there are modified gravity models with no dark energy that are also consistent with the data [2]. Next-generation experiments such as Dark Energy Survey (DES) [3], LSST [4], EUCLID [5], and the SKA [6] are expected to dramatically improve on current constraints and introduce new observables.

It is typical to parametrize $w(z)$ in order to differentiate between various dark energy models, or to parametrize background and perturbation variables to test classes of modified gravity models. A complementary approach is to test the consistency of the concordance model itself, independent of the values of $\Omega_{m}$ and $\Omega_{K}$. A range of null tests designed specifically to probe various aspects of the concordance model have been introduced (see e.g. [7-14] and [15] for a review).

Type Ia supernovae (SNIa) are the best distance indicators to probe the expansion history of the Universe. These "standardizable candles" can be observed to high redshift, and have produced convincing evidence that the Universe has undergone a recent phase of accelerated expansion. Current samples of SNIa (e.g. [16-20]) comprise several hundred SNIa with $z<1.8$. Forthcoming surveys of SNIa, such as DES [21], will produce well-measured light curves for over 4000 SNIa, improving the cosmological constraints by an order of magnitude.
In this paper we use luminosity distances $d_{L}(z)$ determined from SNIa observations to test the consistency of the concordance model, through a set of null tests. Reconstructing the expansion history of the Universe in a model-independent fashion is essential for these tests. To do this, we use Gaussian processes (GP), which have previously been used to reconstruct $w(z)$ from SNIa luminosity distances [22-26]. Our analysis is built on [14], which used $H(z)$ data from the baryon acoustic oscillation (BAO) scale and galaxy ages to test the validity of the concordance model. We use GaPP (Gaussian processes in PYTHON), ${ }^{1}$ a package developed by Seikel and introduced in [25].

The tests based on $H(z)$ are potentially stronger discriminators of the concordance model than those using SNIa data, since the null tests using $d_{L}(z)$ require higher derivative terms than those using $H(z)$. However, null tests based on direct distance measurements currently have the advantage that the data sets are much larger and the errors are smaller.

\section{NULL TESTS OF $\Lambda$ CDM-THEORY}

The Friedmann equation,

$$
\begin{aligned}
\frac{H^{2}(z)}{H_{0}^{2}}= & \Omega_{m}(1+z)^{3}+\Omega_{K}(1+z)^{2} \\
& +\left(1-\Omega_{m}-\Omega_{K}\right) \exp \left[3 \int_{0}^{z} \frac{1+w(z \prime)}{1+z \prime} d z \prime\right],
\end{aligned}
$$

\footnotetext{
${ }^{1}$ http://www.acgc.uct.ac.za/ seikel/GAPP/index.html.
} 
determines the Hubble rate $H$ in terms of today's values for the density parameters for matter $\Omega_{m}$ and curvature $\Omega_{K}$. This is integrated over to obtain the luminosity distances of SNIa:

$$
d_{L}(z)=\frac{(1+z)}{H_{0} \sqrt{-\Omega_{K}}} \sin \left(\sqrt{-\Omega_{K}} \int_{0}^{z} \frac{d z^{\prime}}{H\left(z^{\prime}\right) / H_{0}}\right) .
$$

The equation of state parameter of dark energy, $w=$ $p_{\mathrm{de}} / \rho_{\mathrm{de}}$, can be expressed in terms of the dimensionless comoving luminosity distance,

$$
D(z) \equiv H_{0}(1+z)^{-1} d_{L}(z),
$$

as [27-29]:

$$
\begin{aligned}
w(z)= & \left\{2(1+z)\left(1+\Omega_{K} D^{2}\right) D^{\prime \prime}-\left[(1+z)^{2} \Omega_{K} D^{\prime 2}\right.\right. \\
& \left.\left.+2(1+z) \Omega_{K} D D^{\prime}-3\left(1+\Omega_{K} D^{2}\right)\right] D^{\prime}\right\} / \\
& \left\{3 \left\{(1+z)^{2}\left[\Omega_{K}+(1+z) \Omega_{m}\right] D^{\prime 2}\right.\right. \\
& \left.\left.-\left(1+\Omega_{K} D^{2}\right)\right\} D^{\prime}\right\} .
\end{aligned}
$$

Given an observed distance-redshift relationship $D(z)$, it is possible to reconstruct the equation of state of dark energy and test the $\Lambda \mathrm{CDM}$ model [25]. However, a disadvantage of this method is that it depends on the values of the density parameters, $\Omega_{m}$ and $\Omega_{K}$, which must be measured independently [25].

To avoid this problem and test $\Lambda$ CDM using SNIa data, we use the consistency tests introduced in [9] (see also $[10,11])$. Following this approach, we test the null hypothesis that the expansion of the Universe can be described by a flat or a curved $\Lambda$ CDM model.

The assumptions underlying the consistency tests and the null hypothesis are: (1) the universe is homogeneous and isotropic on large scales; (2) gravity obeys general relativity; (3) the Universe contains cold matter (with $w=0$ ) and dark energy. Photons and neutrinos can be included $\left(\Omega_{\gamma}, \Omega_{\nu}\right.$ are known independently, from CMB data), but it is reasonable to neglect radiation at the low redshifts probed by SNIa data. Detection of a deviation from the consistency tests would imply a violation of at least one of these assumptions: (1) large-scale nonlinear inhomogeneity or anisotropy; (2) modified gravity; (3) dynamical dark energy $(w \neq-1)$, or alternatively, a cosmological constant plus an unknown additional species with equation of state which deviates from that of cold matter, curvature, or vacuum energy. Any of these possibilities imply that the standard $\Lambda \mathrm{CDM}$ is ruled out. Note that the tests cannot identify which of these possibilities applies.

For a flat concordance model, i.e. $w=-1$ and $\Omega_{K}=0$, from (2) we find that

$$
\Omega_{m}\left[(1+z)^{3}-1\right] D^{\prime 2}=1-D^{\prime 2} .
$$

If we define

$$
\mathcal{O}_{m}^{(1)}(z)=\frac{1-D^{\prime}(z)^{2}}{\left[(1+z)^{3}-1\right] D^{\prime}(z)^{2}},
$$

then

$$
\text { flat } \Lambda \text { CDM implies } \mathcal{O}_{m}^{(1)}(z)=\Omega_{m} \text {. }
$$

Thus we obtain a null test of the concordance model:

$$
\mathcal{O}_{m}^{(1)}(z) \neq \Omega_{m} \text { falsifies flat } \Lambda \text { CDM. }
$$

Any variation of $\mathcal{O}_{m}^{(1)}(z)$ with redshift reflects an inconsistency between the flat $\Lambda \mathrm{CDM}$ model and observations. To detect evolution of $\mathcal{O}_{m}^{(1)}$ with redshift we can differentiate $\mathcal{O}_{m}^{(1)}(z)$, from which we define the additional diagnostic:

$$
\begin{aligned}
\mathcal{L}^{(1)}(z)= & (1+z)^{-6}\left\{2\left[(1+z)^{3}-1\right] D^{\prime \prime}(z)\right. \\
& \left.+3(1+z)^{2} D^{\prime}(z)\left[1-D^{\prime}(z)^{2}\right]\right\},
\end{aligned}
$$

which vanishes if and only if $d \mathcal{O}_{m}^{(1)} / d z=0$. The factor $(1+z)^{-6}$ (which was not used in [9]), ensures stability of the errors (see below). If $\mathcal{L}^{(1)}$ is nonzero at any redshift, then observations are incompatible with $\Lambda$ CDM:

$$
\mathcal{L}^{(1)} \neq 0 \text { falsifies flat } \Lambda \mathrm{CDM} .
$$

We can extend this approach to include spatial curvature, and derive null tests for general (curved) $\Lambda$ CDM. Using (1), (2), and (5) with $w(z)=-1$, and solving for $\Omega_{m}$ and $\Omega_{K}$, we find $[12,15]$

$$
\begin{aligned}
\Omega_{m}= & 2 \Upsilon(z)\left\{\left[(1+z)^{2}-D^{2}-1\right] D^{\prime \prime}\right. \\
& \left.-\left(D^{\prime 2}-1\right)\left[(1+z) D^{\prime}-D\right]\right\} \equiv \mathcal{O}_{m}^{(2)}(z),
\end{aligned}
$$

$$
\begin{aligned}
\Omega_{K} & =\Upsilon(z)\left\{2\left[1-(1+z)^{3}\right] D^{\prime \prime}+3 D^{\prime}\left(D^{\prime 2}-1\right)(1+z)^{2}\right\} \\
& \equiv \mathcal{O}_{K}(z) .
\end{aligned}
$$

Here $\Upsilon(z)$ is defined by

$$
\begin{aligned}
\Upsilon^{-1}= & -2\left[1-(1+z)^{3}\right] D^{2} D^{\prime \prime} \\
& -\left\{(1+z)\left[(1+z)^{3}-3(1+z)+2\right] D^{\prime 2}\right. \\
& \left.-2\left[1-(1+z)^{3}\right] D D^{\prime}-3(1+z)^{2} D^{2}\right\} D^{\prime} .
\end{aligned}
$$

Then we have

$$
\mathcal{O}_{m}^{(2)}(z) \neq \Omega_{m} \text { falsifies curved } \Lambda \mathrm{CDM},
$$




$$
\mathcal{O}_{K}^{(2)}(z) \neq \Omega_{K} \text { falsifies curved } \Lambda \text { CDM. }
$$

These are not independent tests: the derivative of $\mathcal{O}_{K}^{(2)}$ vanishes if and only if the derivative of $\mathcal{O}_{m}^{(2)}$ vanishes. Hence, we need only a single diagnostic for vanishing derivative. We use the derivative of $\mathcal{O}_{m}^{(2)}$ to define

$$
\begin{aligned}
\mathcal{L}^{(2)}= & (1+z)^{-6} D^{\prime 2}\{D[-3(1+z) \\
& \times\left(D^{\prime 2}-1\right)\left(2 D^{\prime}+3(1+z) D^{\prime \prime}\right) \\
& \left.+2 z D^{\prime \prime \prime}(3+z(3+z))\right]+9(1+z)^{2} D^{2} D^{\prime \prime 2} \\
& +3(1+z) D^{2} D^{\prime}\left(2 D^{\prime \prime}-(1+z) D^{\prime \prime \prime}\right) \\
& +6(1+z)^{2} D^{\prime 2}\left(D^{\prime 2}-1\right)-\left[3 z^{2}(3+z) D^{\prime \prime 2}\right. \\
& \left.\left.+z D^{\prime}\left(z(3+z) D^{\prime \prime \prime}-6(2+z) D^{\prime \prime}\right)\right](1+z)\right\},
\end{aligned}
$$

which vanishes if and only if $d \mathcal{O}_{m}^{(2)} / d z=0$. (Again we use the prefactor to stabilize the errors.) Then we have the null test for curved $\Lambda \mathrm{CDM}$ :

$$
\mathcal{L}^{(2)}(z) \neq 0 \quad \text { falsifies curved } \Lambda \mathrm{CDM}
$$

In principle, $\mathcal{L}^{(1)}$ and $\mathcal{L}^{(2)}$ provide no additional information compared to $\mathcal{O}_{m}^{(1)}$ and $\mathcal{O}_{m}^{(2)}$. However, it is easier to detect a deviation from zero than to confirm that a quantity is constant, especially since the exact value of this constant is not known a priori. The disadvantage of $\mathcal{L}^{(1)}$ and $\mathcal{L}^{(2)}$ is that they require higher derivatives than $\mathcal{O}_{m}^{(1)}$ and $\mathcal{O}_{m}^{(2)}$, which are more challenging to constrain.

Another problem with $\mathcal{L}^{(1)}$ and $\mathcal{L}^{(2)}$ is the degeneracy between $w$ and $\Omega_{m}$ : a model with redshift dependent $w$ can be formally consistent with $\Lambda \mathrm{CDM}$ within the error bars of the reconstruction if the value of $\Omega_{m}$ is adjusted accordingly. Such cases can only be identified with the $\mathcal{O}_{m}$ tests, but not with $\mathcal{L}$ (see Sec. IV for details).

Note that $\mathcal{L}^{(1)}$ and $\mathcal{L}^{(2)}$ are not identical to $d \mathcal{O}_{m}^{(1)} / d z$ and $d \mathcal{O}_{m}^{(2)} / d z$, respectively. Starting from these two derivatives, we have neglected the denominators, which add significant noise to the tests without adding extra information, and used a prefactor $(1+z)^{-6}$ to obtain $\mathcal{L}^{(1)}$ and $\mathcal{L}^{(2)}$. We are free to do this without loss of generality, since we are testing the equality of these quantities with zero. As a consequence, the error bands of the reconstructions do not necessarily increase with redshift as one might expect, and the size of the errors of $\mathcal{L}^{(1)}$ and $\mathcal{L}^{(2)}$ are not directly comparable. In addition, the errors added from extra redshift factors are small when we have spectroscopic redshift measurements.

\section{NULL TESTS USING SNIA DATA}

To apply these null tests using current data sets, it is essential to choose a model-independent method to reconstruct $D(z)$ and its derivatives. For this purpose, we use GP (via the GaPP code [25]) to smooth the data and reconstruct the derivatives.

\section{A. Gaussian processes}

GP provide a distribution over functions that are suitable to describe the data. At each point $z_{i}$, the distribution of function values $f\left(z_{i}\right)$ is a Gaussian. Thus, the reconstruction consists of a mean function with Gaussian error bands. The function values at different points are correlated by a covariance function $k(z, \tilde{z})$, which depends on a set of hyperparameters (e.g. the characteristic length scale $\ell$ and the signal variance $\sigma_{f}$ ). This also provides a robust way to estimate derivatives of the function in a stable manner.

In contrast to parametric methods, GP do not assume a specific form for the reconstructed function. Instead only typical changes of the function are considered. The hyperparameter $\ell$ corresponds roughly to the distance one needs to move in input space before the function value changes significantly, while $\sigma_{f}$ describes typical changes in the function value.

The choice of covariance function affects the reconstruction to some extent. A general purpose covariance function is the squared exponential covariance function $k(z, \tilde{z})=\sigma_{f}^{2} \exp \left[-(z-\tilde{z})^{2} /\left(2 \ell^{2}\right)\right]$. However, here we use the Matérn $(\nu=9 / 2)$ covariance function:

$$
\begin{aligned}
k(z, \tilde{z})= & \sigma_{f}^{2} \exp \left(-\frac{3|z-\tilde{z}|}{\ell}\right) \\
& \times\left[1+\frac{3|z-\tilde{z}|}{\ell}+\frac{27(z-\tilde{z})^{2}}{7 \ell^{2}}\right. \\
& \left.+\frac{18|z-\tilde{z}|^{3}}{7 \ell^{3}}+\frac{27(z-\tilde{z})^{4}}{35 \ell^{4}}\right] .
\end{aligned}
$$

For a given covariance function, the probability distribution of the hyperparameters depends only on the data. It is necessary either to marginalize over the hyperparameters $\sigma_{f}$ and $\ell$ or to fix the hyperparameters to their maximum likelihood values. Here we choose the latter approach, which is a good approximation and computationally much less expensive than marginalization.

We choose the Matérn $(\nu=9 / 2)$ covariance function because it leads to the most reliable results amongst the covariance functions that we have tested. Here, "reliable" means the following: For various assumed cosmological models and many realizations of mock data sets, the assumed model on average lies within the reconstructed $1-\sigma$ limits for approximately $68 \%$ of the redshift range (and within the reconstructed $2-\sigma$ limits for $\sim 95 \%$ of the redshift range). These values are theoretically expected, thus making Matérn $(\nu=9 / 2)$ a reliable covariance function for our purposes. A detailed analysis regarding the optimal choice of covariance function can be found in [30]. (Note that these results only apply to GP 
reconstructions using $D$ measurements. When applying GP to other data, another covariance function might be more reliable.)

We follow [14,25], which contain a summary of the technical details of GP. The only difference in our approach here is that we use the Matern covariance function (18) instead of the squared exponential. (For detailed reviews of GP, see $[31,32]$.)

\section{B. Application to real data}

We now apply GP to the Union 2.1 data set [17] and determine the current constraints on the consistency of $\Lambda$ CDM. This data set comprises 580 SNIa, with $0.015<z<1.5$, and includes a covariance matrix which incorporates a systematic uncertainty.

The distance modulus, $\mu=m-M$, is the difference between the observed magnitude $m(z)$ and the absolute magnitude of an object $M$, and is given by

$$
\mu(z)+5 \log H_{0}-25=5 \log [(1+z) D(z)] .
$$

We choose $H_{0}=70 \mathrm{kms}^{-1} \mathrm{Mpc}^{-1}$, as in [17]. Note that $H_{0}$ and $M$ are degenerate in (19) so we can fix $H_{0}$ and only consider the uncertainties in $M$ which are included in the covariance matrix of the Union 2.1 data set [17] - this includes the errors on $H_{0}$. We convert $\mu$ to $D$ and add the
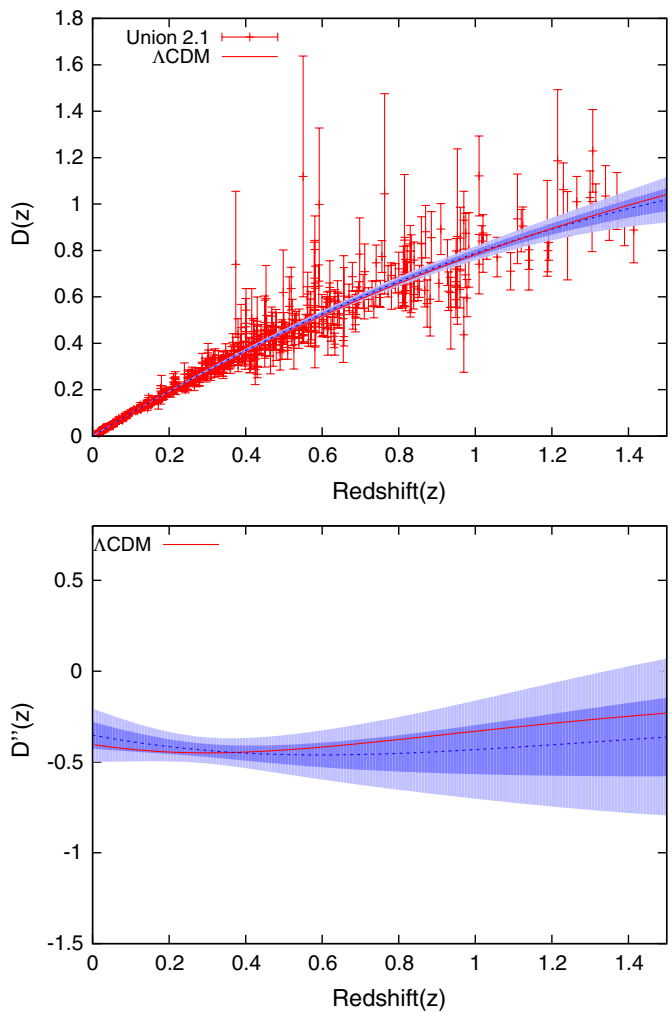

theoretical values $D(z=0)=0$ and $D^{\prime}(z=0)=1$ to the data set.

Figure 1 shows the reconstructed $D(z)$ and its first three derivatives for the Union 2.1 data set, while Fig. 2 shows the inferred reconstructions for $\mathcal{O}_{m}^{(1)}, \mathcal{O}_{m}^{(2)}$ and $\mathcal{O}_{K}^{(2)}$. Figure 3 shows the reconstruction of $\mathcal{L}^{(1)}$ and $\mathcal{L}^{(2)}$.

The errors on the reconstructed distances in Fig. 1 increase with increasing order of derivative. For example, at $z=1.5$, the standard deviation is 0.05 for the reconstruction of $D, 0.12$ for $D^{\prime}, 0.22$ for $D^{\prime \prime}$, and 0.29 for $D^{\prime \prime \prime}$. The near constancy of the errors on $D^{\prime \prime \prime}$ reflects the fact that we are unable to constrain rapid variations (carried via higher derivatives) on scales below a typical length scale, which is roughly associated with $\ell$. By using Gaussian processes the scale $\ell$ and the resulting smoothness of the reconstruction is driven purely by the data. Where there is insufficient evidence for rapid variations, a smooth function will result, which we see in the second and third derivatives. Further analysis of the redshift dependence of the errors can be found in the Appendix.

\section{Mock data}

To demonstrate the ability of the null tests to distinguish between different cosmological models when applied to future SNIa data sets, we produce mock catalogues for two fiducial models:
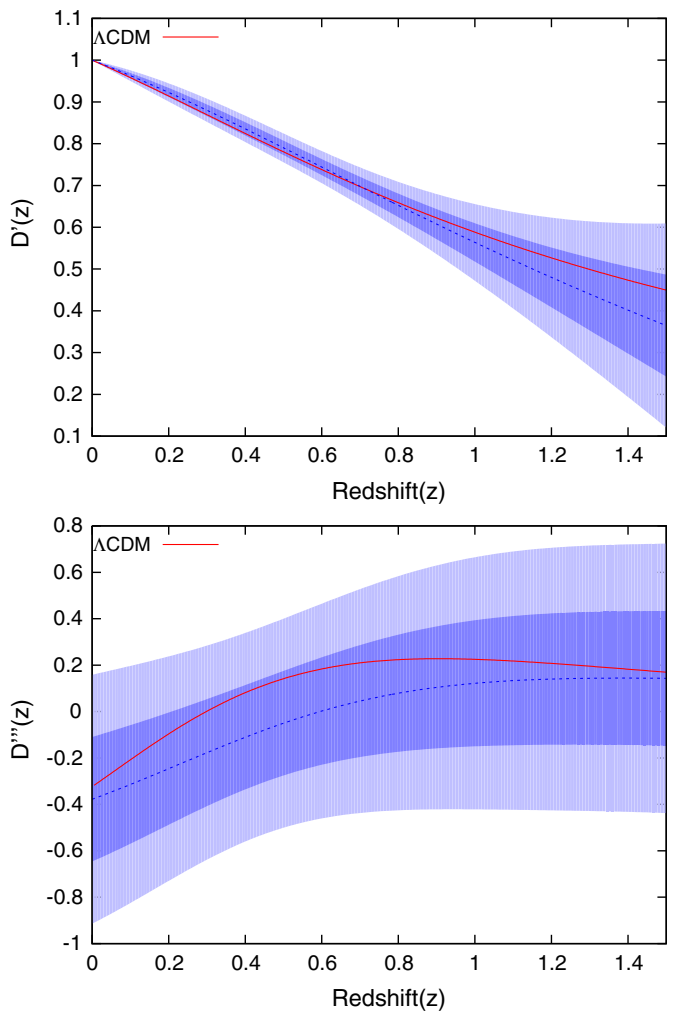

FIG. 1 (color online). Gaussian processes reconstruction of $D, D^{\prime}$ (top) and $D^{\prime \prime}, D^{\prime \prime \prime}$ (bottom) for Union 2.1 data. The red (solid) line is flat $\Lambda$ CDM with $\Omega_{m}=0.27$. The blue (dashed) line is the mean of the reconstruction. Shaded areas give 95\% (light) and $68 \%$ (dark) confidence limits of the reconstructed function. 
NULL TESTS OF THE COSMOLOGICAL CONSTANT USING ...

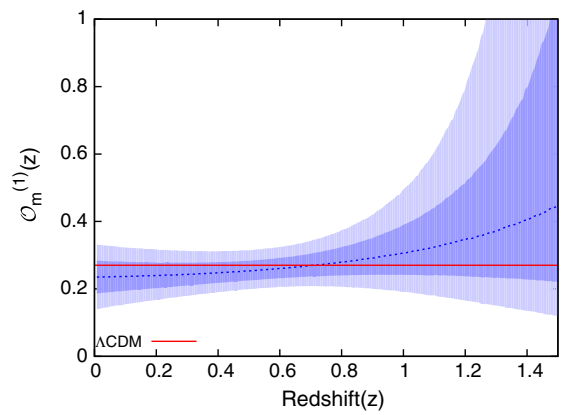

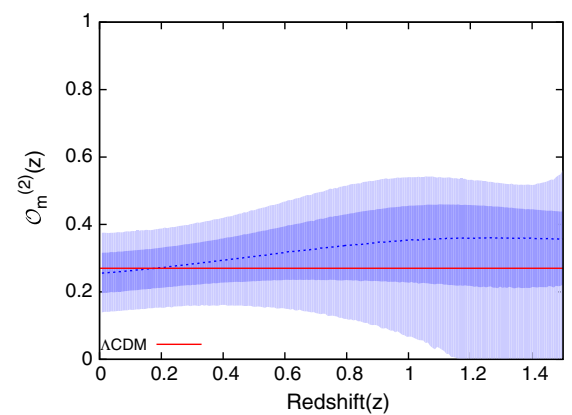

PHYSICAL REVIEW D 89, 023503 (2014)

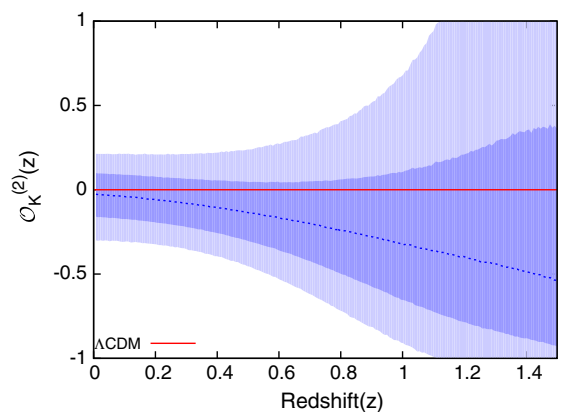

FIG. 2 (color online). Reconstruction of $\mathcal{O}_{m}^{(1)}$ (left), $\mathcal{O}_{m}^{(2)}$ (middle) and $\mathcal{O}_{K}^{(2)}$ (right) for Union 2.1 data. Lines and shadings are as in Fig. 1.
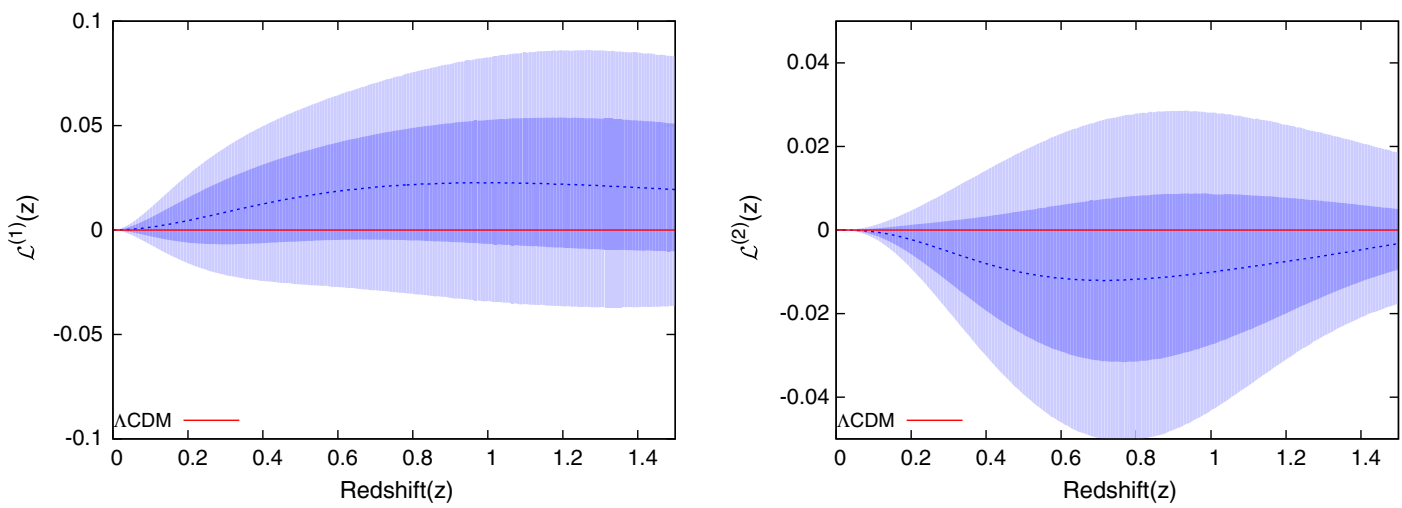

FIG. 3 (color online). Reconstruction of $\mathcal{L}^{(1)}$ (left) and $\mathcal{L}^{(2)}$ (right) for Union 2.1 data. Lines and shadings are as in Fig. 1.
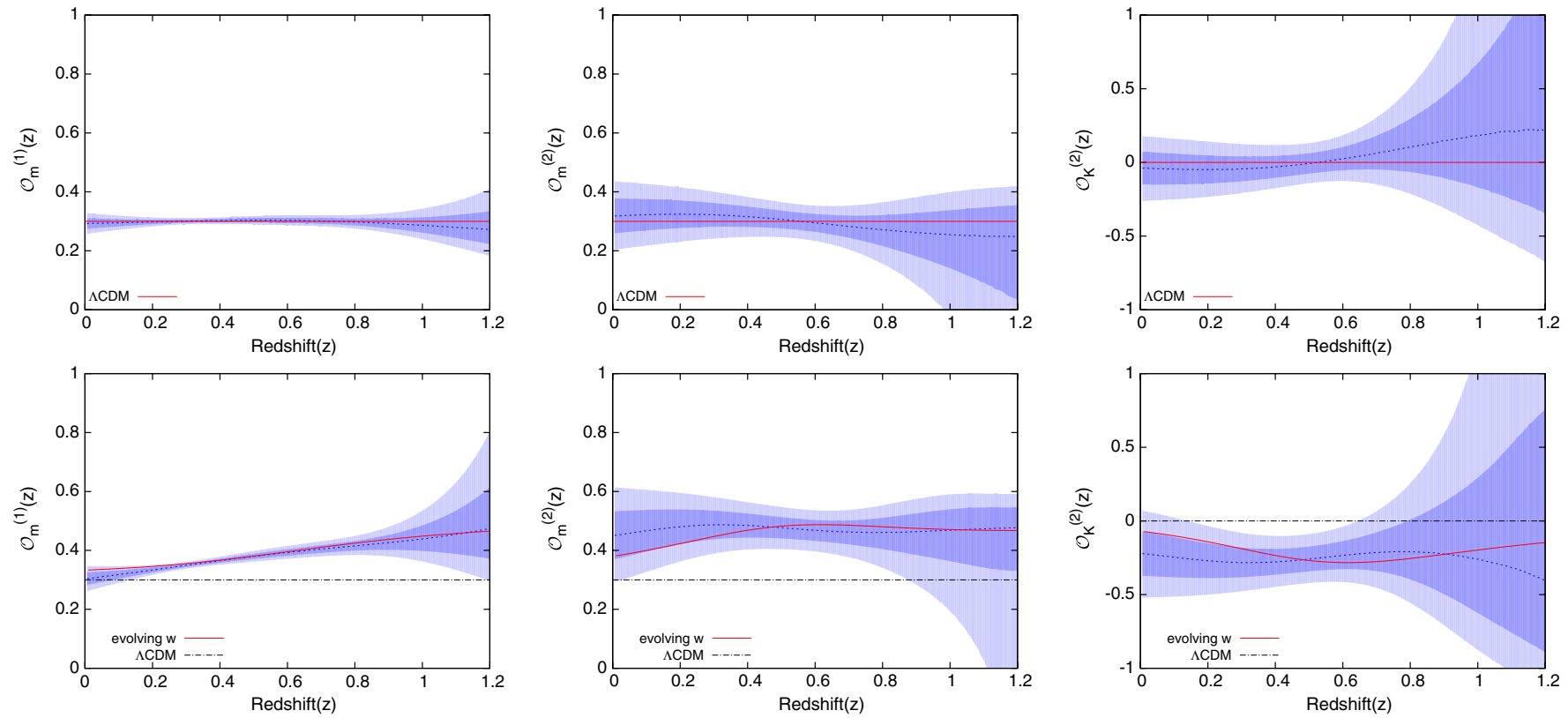

FIG. 4 (color online). $\quad \mathcal{O}_{m}^{(1)}$ (left), $\mathcal{O}_{m}^{(2)}$ (middle), and $\mathcal{O}_{K}^{(2)}$ (right) reconstructed using simulated DES data, and assuming $\Lambda$ CDM (top) and the evolving $w$ in (20) (bottom). 

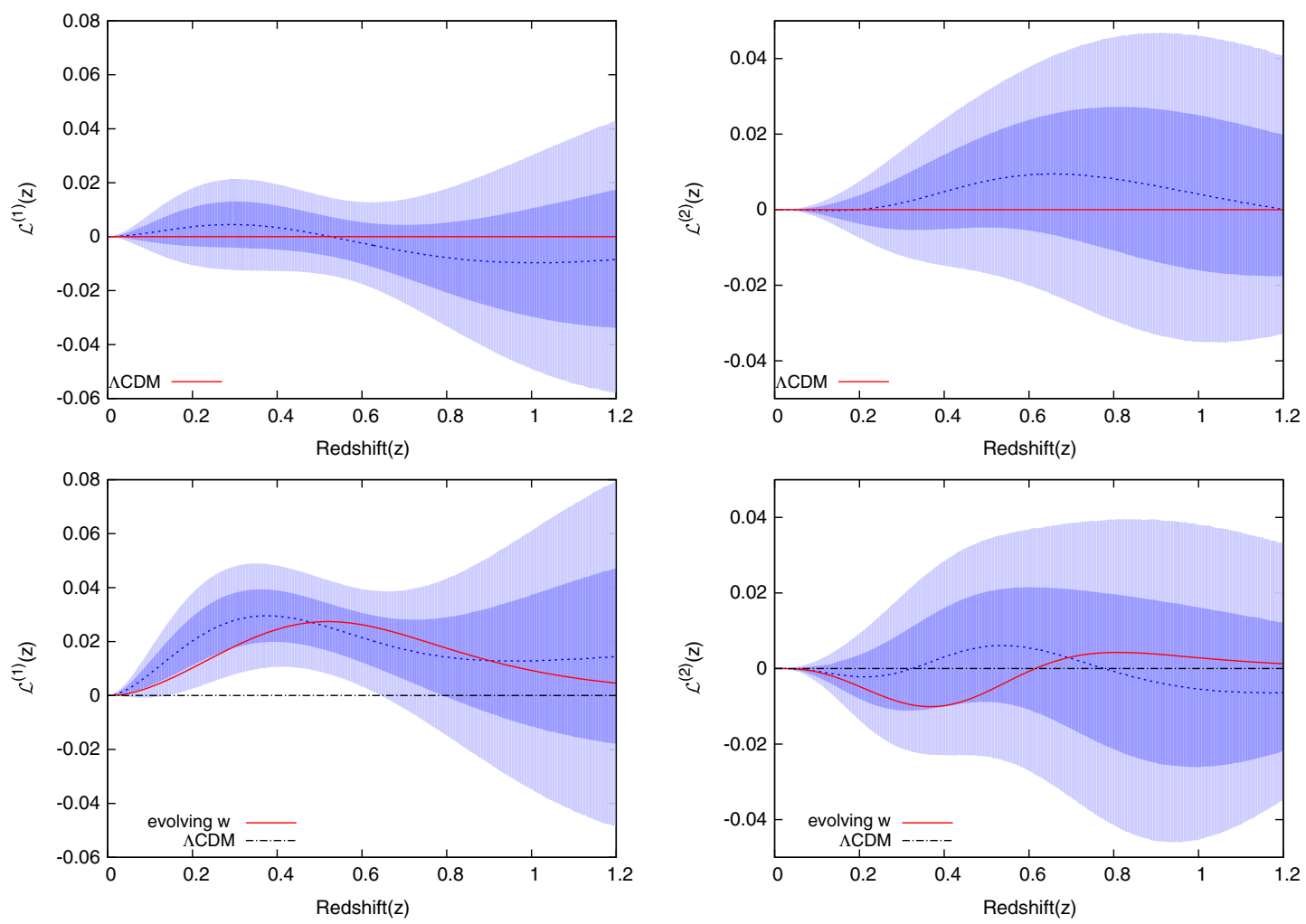

FIG. 5 (color online). Reconstruction of $\mathcal{L}^{(1)}$ (left) and $\mathcal{L}^{(2)}$ (right) for simulated DES data, and assuming $\Lambda$ CDM (top) and the evolving $w$ in (20) (bottom). Due to the degeneracy between $w$ and $\Omega_{m}$, the reconstruction of $\mathcal{L}^{(2)}$ for the model with evolving dark energy is consistent with $\Lambda$ CDM. However, the inferred values of $\Omega_{m}$ and $\Omega_{K}$ differ significantly from the input value as can be seen in Fig. 4.

(i) flat $\Lambda \mathrm{CDM}$

(ii) dynamical dark energy model with $\Omega_{K}=0$ and

$$
w(z)=\frac{1}{2}\left\{-1+\tanh \left[3\left(z-\frac{1}{2}\right)\right]\right\} .
$$

We take $\Omega_{m}=0.3$. Using the redshift distribution and scatter anticipated by the Dark Energy Survey (DES) [21], we simulate $\sim 4000$ data points in the redshift range $0<z<1.2$. Note that the scatter only includes statistical errors.

For each of the two simulated data sets, we reconstruct $D(z)$ and its derivatives and apply the null tests. Figure 4 shows the constraints and uncertainties on $\mathcal{O}_{m}^{(1)}, \mathcal{O}_{m}^{(2)}$, and $\mathcal{O}_{K}^{(2)}$ for both models, while Fig. 5 shows the results for $\mathcal{L}^{(1)}$ and $\mathcal{L}^{(2)}$.

\section{DISCUSSION}

We have introduced an approach to applying null tests of the $\Lambda$ CDM models (flat and curved). Using a GP technique to reconstruct the distance-redshift relationship and its derivatives from SNIa data sets in a model-independent fashion, we have shown that the flat concordance model is consistent with current data, falling within the $1 \sigma$ limits. The null tests are stronger if we assume flatness, as expected.

For the Union 2.1 data set, the consistency tests are in good agreement with a constant, indicating no evidence of a deviation from a flat $\Lambda \mathrm{CDM}$ model (see Figs. 2 and 3). For the $\mathcal{O}_{m}^{(1)}$ and $\mathcal{O}_{m}^{(2)}$ tests we find a value for $\Omega_{m} \sim 0.27$. $\mathcal{O}_{K}^{(2)}$ is consistent with zero, as expected for flat $\Lambda \mathrm{CDM}$. Due to the limited number of SNIa in the Union 2.1 sample and the model-independent method we use, the reconstructed uncertainties are significant.

For a mock data set based on the DES supernova survey, we find that our approach can distinguish between competing cosmological models. Using a simulated sample drawn from a flat $\Lambda \mathrm{CDM}$ model, the recovered distribution of $\mathcal{O}_{m}^{(1)}$ is constant over the redshift range considered (Fig. 4), consistent with $\mathcal{O}_{m}^{(1)}=\Omega_{m}$. For the evolving $w$ model of (20), $\mathcal{O}_{m}^{(1)}$ deviates strongly from a constant value, so that flat $\Lambda \mathrm{CDM}$ would be disfavored. This is confirmed by the deviation of $\mathcal{L}^{(1)}$ from zero in Fig. 5.

When spatial curvature is allowed, the constraints from the null tests tend to be weakened, as would be expected by the degeneracy introduced by the extra degree of freedom [33]. For a flat $\Lambda \mathrm{CDM}$ fiducial model, the reconstructed distributions of $\mathcal{O}_{m}^{(2)}$ and $\mathcal{O}_{K}^{(2)}$ are consistent with being 
constant and equal to $\Omega_{m}$ and $\Omega_{K}$ (Fig. 4), respectively, confirming that the model does not deviate from $\Lambda \mathrm{CDM}$, as anticipated. But the errors are significantly larger when curvature is allowed.

For the evolving $w$ fiducial model, the reconstructions of $\mathcal{O}_{m}^{(2)}$ and $\mathcal{O}_{K}^{(2)}$ are consistent with constants (Fig. 4)-but these constant values differ significantly from the input values of $\Omega_{m}$ and $\Omega_{K}$, respectively. The evolving $w$ model can erroneously be interpreted as a $\Lambda$ CDM model with a large matter density $\Omega_{m}$ and negative curvature $\Omega_{K}$. Consequently, the reconstruction of $\mathcal{L}^{(2)}$ (Fig. 5) is consistent with a constant, indicating that $\Lambda \mathrm{CDM}$ is not disfavored. In both cases, the errors are large and the null tests are degraded.

This problem reflects the degeneracy between the density parameters and the dark energy equation of state (see also $[25,33,34])$. The reconstructions are formally consistent with a constant, and thus with $\Lambda \mathrm{CDM}$, due to their incorrectly inferred values. Additional constraints on the value of $\Omega_{m}$ and $\Omega_{K}$ from, for instance, BAO or CMB measurements, are needed to break this degeneracy.

\section{CONCLUSIONS}

In this paper, we described a series of null tests that can be applied to SNIa data to determine the consistency of observations with a (flat) $\Lambda \mathrm{CDM}$ model-without the need to parametrize the equation of state of dark energy. The tests require that the distance $D$ and the diagnostics $\mathcal{O}_{m}^{(1)}, \mathcal{O}_{m}^{(2)}$, $\mathcal{O}_{K}^{(2)}, \mathcal{L}^{(1)}$, and $\mathcal{L}^{(2)}$ are reconstructed in a model-independent way. We used GP to perform these reconstructions.

We applied the null tests to the Union 2.1 SNIa data set. The results were consistent with a flat $\Lambda \mathrm{CDM}$ model (Figs. 2 and 3).

Using the anticipated redshift distribution for the DES supernova survey, we produced mock data sets of 4000 SNIa, with two competing fiducial cosmological models: flat $\Lambda \mathrm{CDM}$ and an evolving $w$ model. The reconstructed distributions of $\mathcal{O}_{m}^{(1)}$ for these data sets show that the consistency tests are able to distinguish between different cosmological models, and can correctly identify deviations from $\Lambda \mathrm{CDM}$, in the case when spatial flatness is assumed. However, allowing for spatial curvature degrades the null tests in general (although not always - see Fig. 2). The inherent degeneracy between the equation of state of dark energy and the density parameters $\left(\Omega_{m}, \Omega_{K}\right)$ reduces our ability to distinguish between various models. The distributions of $\mathcal{O}_{m}^{(2)}$, $\mathcal{O}_{K}^{(2)}$, and $\mathcal{L}^{(2)}$ were consistent with a constant for the evolving $w$ model (Fig. 5), but the inferred values of $\Omega_{m}$ and $\Omega_{K}$ from the $\mathcal{O}_{m}^{(2)}$ and $\mathcal{O}_{K}^{(2)}$ distributions were unrealistic (Fig. 4). The degeneracy needs to be broken using other data.

For future data sets which will have the power to probe $\Lambda \mathrm{CDM}$ at high precision, the null tests we have introduced will require further refinement. In particular, we need to develop a method of quantifying the significance of any possible deviation. This is left for future work.

\section{ACKNOWLEDGMENTS}

We thank Heather Campbell for helpful discussions on SNIa data. S. Y., M. S., and R. M. were supported by the South Africa Square Kilometre Array Project and the South African National Research Foundation (NRF). C. C. was supported by the NRF. R. M. is supported by the UK Science \& Technology Facilities Council (Grants No. ST/ H002774/1 and No. ST/K0090X/1). All authors were supported by a Royal Society (UK)/National Research Foundation (SA) exchange grant. S. Y. was supported by the DAAD (German Academic Exchange Service).

\section{APPENDIX: REDSHIFT DEPENDENCE OF THE ERRORS ON THE GP RECONSTRUCTIONS}

The error of the GP reconstruction of the $n$th derivative on $D$ at point $z^{*}$ is given by

$$
\begin{aligned}
\sigma\left(D^{(n)}\left(z^{*}\right)\right)= & \left(k^{(n, n)}\left(z^{*}, z^{*}\right)\right. \\
& \left.-K^{(n, 0)}\left(z^{*}, \boldsymbol{Z}\right)[K(\boldsymbol{Z}, \boldsymbol{Z})+C]^{-1} K^{(0, n)}\left(\boldsymbol{Z}, z^{*}\right)\right)^{\frac{1}{2}} .
\end{aligned}
$$

Here, $\boldsymbol{Z}$ is a vector containing the locations $z_{i}$ of the data and $C$ is the covariance matrix of the data. $k$ denotes the covariance function [here, Matérn $(\nu=9 / 2)$ as given by Eq. (18)] and $K$ a matrix containing covariances between the redshift points: $[K(\boldsymbol{Z}, \boldsymbol{Z})]_{i j}=k\left(z_{i}, z_{j}\right)$. Taking the $n$th derivative of $k$ with respect to the first argument and the $m$ th derivative with respect to the second argument is denoted as $k^{(n, m)}$.

Note that the first term in the equations for the errors is constant for a given covariance function and hyperparameters. Stationary covariance functions $k\left(z_{i}, z_{j}\right)$, such as Matérn $(\nu=9 / 2)$, only depend on $\left|z_{i}-z_{j}\right|$, but not on $z_{i}$ and $z_{j}$ individually. Therefore, $k^{(n, n)}\left(z^{*}, z^{*}\right)$ does not depend on the value of $z^{*}$.

We rewrite the equation for the errors (A1) as

$$
\sigma\left(D^{(n)}\left(z^{*}\right)\right)=\sqrt{t_{1, n}-t_{2, n}\left(z^{*}\right)},
$$

where

$$
\begin{aligned}
t_{1, n} & =k^{(n, n)}\left(z^{*}, z^{*}\right), \\
t_{2, n}\left(z^{*}\right) & =K^{(n, 0)}\left(z^{*}, \boldsymbol{Z}\right)[K(\boldsymbol{Z}, \boldsymbol{Z})+C]^{-1} K^{(0, n)}\left(\boldsymbol{Z}, z^{*}\right) .
\end{aligned}
$$

$t_{1, n}$ is determined by the covariance function and the hyperparameters. It is constant in redshift and does not explicitly depend on the data. (Note that the data are used to optimize the hyperparameters and thus indirectly affect the value of $t_{1, n}$.) $t_{2, n}\left(z^{*}\right)$ is redshift dependent and also depends on the position and covariance matrix of the data.

Figure 6 shows $t_{1}$ and $t_{2}\left(z^{*}\right)$ for the reconstructions of $D$ and its derivatives. For $D, D^{\prime}$, and $D^{\prime \prime}$, we observe strong 


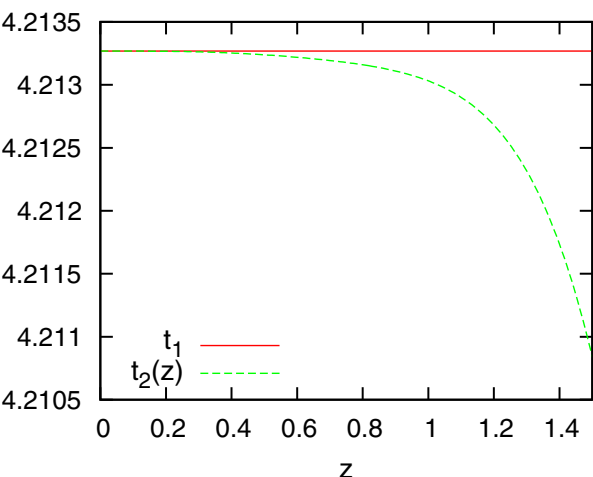

(a)

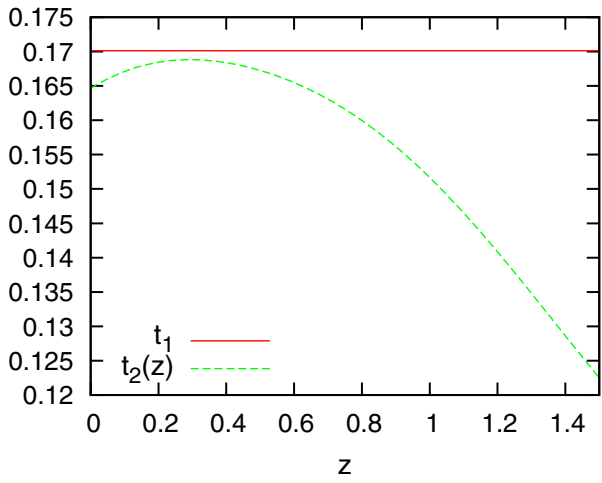

(c)

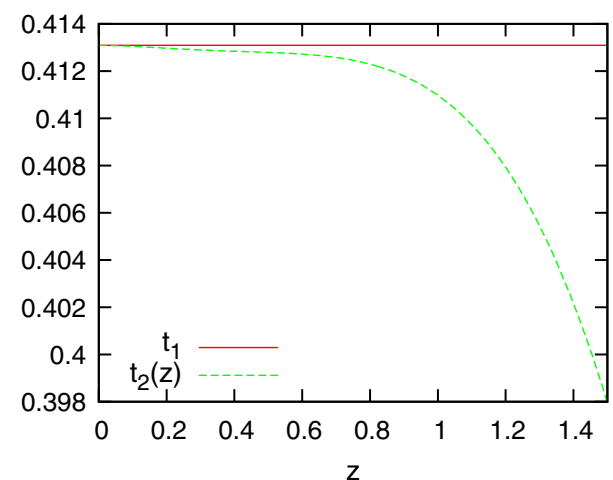

(b)

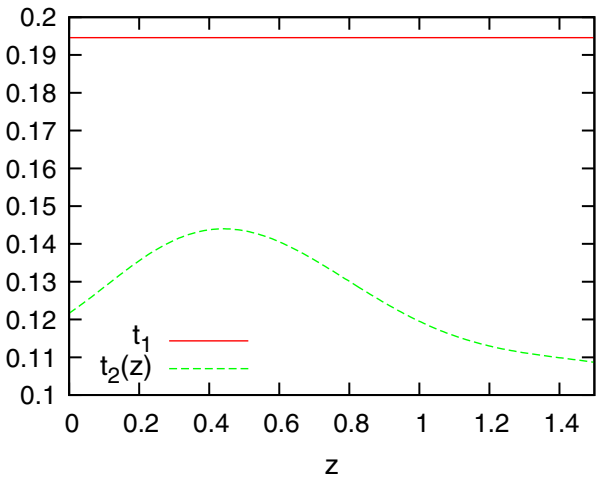

(d)

FIG. 6 (color online). Terms contributing to the calculation of the errors for the GP reconstructions of $D(z), D^{\prime}(z), D^{\prime \prime}(z)$, and $D^{\prime \prime \prime}(z)$. $t_{1}$ and $t_{2}(z)$ are the constant and data dependent terms, respectively, as given by Eq. (A2). (a) $D(z)$. (b) $D^{\prime}(z)$. (c) $D^{\prime \prime}(z)$. (d) $D^{\prime \prime \prime}(z)$

relative changes in $t_{1}-t_{2}\left(z^{*}\right)$ with redshift. We denote the maximum value of this term within the considered redshift range as $\left\{t_{1}-t_{2}\left(z^{*}\right)\right\}_{\max }$ and the mean value as $\left\{t_{1}-t_{2}\left(z^{*}\right)\right\}_{\text {mean }}$. Then we can quantify the redshift dependence of $t_{1}-t_{2}\left(z^{*}\right)$ by the relative variation $v_{r}=\left\{t_{1}-t_{2}\left(z^{*}\right)\right\}_{\max } /\left\{t_{1}-t_{2}\left(z^{*}\right)\right\}_{\text {mean }} . \quad v_{r}=1$ implies that the errors of the reconstruction are constant, while a large value would indicate strong redshift dependence.

We find the following results for $v_{r}$ :

$$
\begin{array}{cc}
v_{r}(D)=6.9, & v_{r}\left(D^{\prime}\right)=5.6, \\
v_{r}\left(D^{\prime \prime}\right)=3.2, & v_{r}\left(D^{\prime \prime \prime}\right)=1.3 .
\end{array}
$$

The values for $D, D^{\prime}$, and $D^{\prime \prime}$ are much larger than 1, implying a strong redshift dependence of the errors. However, for the reconstruction of $D^{\prime \prime \prime}$ the relative variation is much smaller, namely $v_{r}=1.3$. The smallness of this value can be understood by the following consideration: $t_{1} \gg$ $t_{2}\left(z^{*}\right) \forall z^{*}$ ensures that the absolute variations of $t_{1}-$ $t_{2}\left(z^{*}\right)$ (which are large compared to those for the lower derivatives of $D$ ) translate into small relative variations. Therefore, the dominance of the constant term $t_{1}$ is the main reason for the small value of $v_{r}$ and thus the near constancy of the errors on $D^{\prime \prime \prime}$.
[1] P. A. R. Ade et al. (Planck Collaboration), arXiv:1303.5076.

[2] T. Clifton, P. G. Ferreira, A. Padilla, and C. Skordis, Phys. Rep. 513, 1 (2012).

[3] T. Abbott et al. (DES Collaboration), arXiv:astro-ph/0510346.

[4] P. A. Abell et al. (LSST Collaboration), arXiv:0912.0201.

[5] R. Laureijs et al. (EUCLID Collaboration), arXiv:1110.3193.

[6] C. A. Blake, F. B. Abdalla, S. L. Bridle, and S. Rawlings, New Astron. Rev. 48, 1063 (2004).
[7] C. Clarkson, B. Bassett, and T. H.-C. Lu, Phys. Rev. Lett. 101, 011301 (2008).

[8] J.-P. Uzan, C. Clarkson, and G. F. R. Ellis, Phys. Rev. Lett. 100, 191303 (2008).

[9] C. Zunckel and C. Clarkson, Phys. Rev. Lett. 101, 181301 (2008).

[10] V. Sahni, A. Shafieloo, and A. A. Starobinsky, Phys. Rev. D 78, 103502 (2008). 
[11] A. Shafieloo and C. Clarkson, Phys. Rev. D 81, 083537 (2010).

[12] C. Clarkson, AIP Conf. Proc. 1241, 784 (2010).

[13] S. Nesseris and A. Shafieloo, Mon. Not. R. Astron. Soc. 408, 1879 (2010).

[14] M. Seikel, S. Yahya, R. Maartens, and C. Clarkson, Phys. Rev. D 86, 083001 (2012).

[15] C. Clarkson, C.R. Phys. 13, 682 (2012).

[16] A. Conley, J. Guy, M. Sullivan, N. Regnault, P. Astier, C. Balland et al., Astrophys. J. Suppl. Ser. 192, 1 (2011).

[17] N. Suzuki, D. Rubin, C. Lidman, G. Aldering, R. Amanullah, K. Barbary et al., Astrophys. J. 746, 85 (2012).

[18] H. Campbell, C. B. D’Andrea, R. C. Nichol, M. Sako, M. Smith, H. Lampeitl et al., Astrophys. J. 763, 88 (2013).

[19] D. Scolnic, A. Rest, A. Riess, M. E. Huber, R. J. Foley, D. Brout, R. Chornock, G. Narayan et al., arXiv:1310.3824.

[20] A. Rest, D. Scolnic, R. J. Foley, M. E. Huber, R. Chornock, G. Narayan, J. L. Tonry, E. Berger et al., arXiv:1310.3828.

[21] J. P. Bernstein, R. Kessler, S. Kuhlmann, R. Biswas, E. Kovacs, G. Aldering et al., Astrophys. J. 753, 152 (2012).

[22] T. Holsclaw, U. Alam, B. Sanso, H. Lee, K. Heitmann, S. Habib, and D. Higdon, Phys. Rev. D 82, 103502 (2010).
[23] T. Holsclaw, U. Alam, B. Sanso, H. Lee, K. Heitmann, S. Habib, and D. Higdon, Phys. Rev. Lett. 105, 241302 (2010).

[24] T. Holsclaw, U. Alam, B. Sanso, H. Lee, K. Heitmann, S. Habib, and D. Higdon, Phys. Rev. D 84, 083501 (2011).

[25] M. Seikel, C. Clarkson, and M. Smith, J. Cosmol. Astropart. Phys. 06 (2012) 036.

[26] A. Shafieloo, A. G. Kim, and E. V. Linder, Phys. Rev. D 85, 123530 (2012).

[27] A. A. Starobinsky, JETP Lett. 68, 757 (1998).

[28] T. Nakamura and T. Chiba, Mon. Not. R. Astron. Soc. 306, 696 (1999).

[29] D. Huterer and M. S. Turner, Phys. Rev. D 60, 081301 (1999).

[30] M. Seikel and C. Clarkson, arXiv:1311.6678.

[31] C. Rasmussen and C. Williams, Gaussian Processes for Machine Learning (MIT Press, Cambridge, MA, 2006).

[32] D. MacKay, Information Theory, Inference and Learning Algorithms (Cambridge University Press, Cambridge, England, 2003).

[33] C. Clarkson, M. Cortes, and B. A. Bassett, J. Cosmol. Astropart. Phys. 08 (2007) 011.

[34] R. Hlozek, M. Cortes, C. Clarkson, and B. Bassett, Gen. Relativ. Gravit. 40, 285 (2008). 